\title{
Theory and development of magnetic flux leakage sensor for flaws detection: A review
}

\author{
Nor Afandi Sharif ${ }^{1}$, Rizauddin Ramli ${ }^{2}$, Abdullah Zawawi Mohamed ${ }^{3}$, Mohd Zaki Nuawi ${ }^{4}$ \\ ${ }^{1,2,4}$ Centre for Materials Engineering and Smart Manufacturing, Universiti Kebangsaan Malaysia, Malaysia \\ ${ }^{1}$ Department of Industrial Electronic, German Malaysian Institute, Malaysia \\ ${ }^{3}$ PETRONAS Research Sdn Bhd, Malaysia
}

\begin{tabular}{l}
\hline \hline Article Info \\
\hline Article history: \\
Received Apr 29, 2019 \\
Revised Jul 1, 2019 \\
Accepted Jul 16, 2019 \\
\hline
\end{tabular}

Keywords:

Artificial neural network Fenite element method Magnetic flux leakage Non-destructive test

\begin{abstract}
This paper presents a review of state-of-art in the Magnetic Flux Leakage (MFL) sensor technology, which plays an important role in Nondestructive Testing (NDT) to detect crack and corrosion in ferromagnetic material. The demand of more reliable MFL tools and signal acquisition increase as it has a direct impact on structure integrity and can lead to be major catastrophic upon questionable signal analysis. This is because the size, cost, efficiency, and reliability of the extensive MFL system for NDT applications primarily depend on signal acquisition as a qualitative measure in producing a trustworthy analysis. Therefore, the selection of appropriate tools and methodology plays a major role in determining the comprehensive performance of the system. This paper also reviews an Artificial Neural Network (ANN) and Finite Element Method (FEM) in developing an optimum permeability standard on the test piece.
\end{abstract}

Copyright $@ 2019$ Institute of Advanced Engineering and Science. All rights reserved.

\section{Corresponding Author:}

Rizauddin Ramli,

Centre for Materials Engineering and Smart Manufacturing,

Universiti Kebangsaan Malaysia,

43600 UKM Bangi, Selangor, Malaysia.

Email: rizauddin@ukm.edu.my

\section{INTRODUCTION}

The Nondestructive testing (NDT) technique is a popular method used by many industries, especially in oil and gas (O\&G) and as well as in refinery plants $[1,2]$. There are many varieties of NDT technique used in industries, which largely depends upon the methods of their applications [3, 4]. Therefore, determining the quality and integrity of the material is the main purpose on NDT without affecting the capability to perform their intentional functions. In order to select the suitable NDT technique, several basic factors need to be considered such as the product diameter, length, wall thickness, fabrication methods, types, location of potential discontinuities and specification requirements. Furthermore, extraneous variables such as a scratch and oxidation that might cause a rejectable indication, even though the product is acceptable is also an important aspect [5-7].

The emerging technology in NDT has triggered a challenge for the new researchers on meeting the demand of more rapid and accurate data requisition, which saw them progressively developing a modern method to increase its reliability. Several NDT processes such as ultrasonic, radiographic, Magnetic Flux Leakage (MFL) and Eddy currents are the NDT method uses to investigate large structure, piping and tank [8]. Currently, petroleum and chemical industries are the main sectors which employ a tank bottom as storage before the secondary process can be established. Most of the storage tanks are built on top of the ground and exposed to the external environment such as humidity and physical damages [9]. As a result, it will lead to the inter-granular attack and causes severe corrosion and deformation on the tank structure. A fatal accident such as an explosion and major leaking will occur because of a small defect at the tank 
bottom. Usually, the storage tanks in petroleum refineries and heavy industries stores hazardous and flammable chemical. Hence, a minor defect may result a catastrophic accident in term of casualties, production interruption and property loss [10].

Among all the NDT methods, the MFL inspection is one of the most reliable methods in oil and gas industries in producing a credible and prompt result and analysis [11, 12]. It has been used as early as 1868 by Institute of Naval Architecture in England in inspecting a cannon tube with compass. The theories behind the MFL area inspection are similar to the principle of Magnetic Particle Inspection (MPI) except for the sensor used between these two methods. The assessment of MFL technique is depended on evaluating the surface of the specimen which magnetised near to the saturation point before the specimen condition can be viewed based on measuring the magnetic leakage field [13]. The MFL sensor detects volumetric changes of the leakage field on the corrosion spot [14]. According to Mix [15], the permeability of magnetized pieces changed drastically, and leakage flux will emanate from the discontinuity. Hence, by measuring the intensity of the flux leakage, severity and condition of the defect can be determined. Pipe, rod, storage tank plates are the types of ferromagnetic parts that have been widely tested by MFL. In general, there are two types of defects existing in the service of the storage tank which are the corrosion and groove. Therefore, it is important to classify the pattern of the defect in order to evaluate the safety of the components [16].

\section{METHODOLOGY FOR LITERATURE SEARCH}

This paper addressed a review of previous research related to the theory and development of MFL sensor in plate and pipe detection by NDT method. However, issue related to different kinds of sensors in MFL signal is not considered in this paper. All the papers in the research and commercialization stages were collected from SCOPUS, the SCI web and company websites, to which the searches were limited to the period between the years of 1999 to 2016.

\section{TYPES OF MFL SENSORS}

There are many types of MFL sensors in magnetic signal detection such as Hall sensor, superconducting quantum interference device (SQUID) and giant magnet resistance (GMR) [17-19]. The mechanism of Hall Effect sensor is by detecting the change of magnetic field and converts into an electrical signal by processing a raw data into voltage [20]. By using Hall component's sensor, defect information can be acquired by catching the faulty of leakage and process the electrical signal transformed from the magnetic field. Magnetic field distribute homogenously by passing the ferromagnetic material in the absence of defect. If the sensor detects the presence of the imperfection on the surface of the material, the magnetic field passing this area will be distorted. As a result, the resistance around the defect will increase and expose the magnetic leakage at the defective area [21]. There also several sensors in MFL testing that have been introduced to the Non-Destructive Evaluation (NDE) such as fluxgate [22-24], Giant Magneto Impedance (GMI) $[25,26]$ and Stress Impedance (SI) $[27,28]$. Table 1 shows the comparison of MFL types of sensors.

Table 1. Magnetic sensor comparison [29]

\begin{tabular}{lllll}
\hline Sensor & Head length & Resolution & Response speed & Power consumption \\
\hline Hall sensor & $10-100 \mu \mathrm{m}$ & $0.5 \mathrm{Oe} / \pm 1 \mathrm{kOe}$ & $1 \mathrm{MHz}$ & $10 \mathrm{~mW}$ \\
GMR sensor & $10-100 \mu \mathrm{m}$ & $0.01 \mathrm{Oe} / \pm 20 \mathrm{Oe}$ & $1 \mathrm{MHz}$ & $10 \mathrm{~mW}$ \\
Fluxgate & $10-20 \mathrm{~mm}$ & $1 \mu \mathrm{Oe} / \pm 3 \mathrm{Oe}$ & $5 \mathrm{kHz}$ & $1 \mathrm{~W}$ \\
SI sensor & $1-2 \mathrm{~mm}$ & $0.1 \mathrm{Gal} / 30 \mathrm{Gal}$ & $10 \mathrm{kHz}$ & $5 \mathrm{~mW}$ \\
\hline
\end{tabular}

\subsection{Hall sensor}

Hall sensor is constructed according to the Hall-effect principle by embedding a thin electric conductor in sensing a magnetic field fluctuation in a ferromagnetic material $[30,31]$. An electrical current will flow through the strip when the magnetic field is perpendicular to the thin strip of conducting material $[32,33]$. Hall sensor has been proved of having a high sensitivity in detecting defect in ferro magnetic material using direct current (DC) as an excitation [34]. The hall voltage can be represented as

$$
V_{h}=\frac{I B}{n e b}
$$

Where $V_{h}, I, B$, ne and $b$ represent the Hall voltage, impose current, magnetic induction intensity, Hall element sensitivity and the thickness of the hall element, respectively [35]. By substituting the Hall element sensitivity, $K_{h}=(\text { neb })^{-1}$ in to (1), the equation can be shown as follows

Theory and development of magnetic flux leakage sensor for flaws detection: A review (Nor Afandi Sharif) 
$B=\frac{V_{h} K_{h}}{I}$

The (2) shows the linear relationship between the magnetic flux density and the hall voltage. The output voltage of the sensor is directly proportional to the flex density. The sensitivity of the Hall sensor's base silicon is $1 \mathrm{mV} / \mathrm{mT}$ for a $1 \mathrm{~mA}$ current and Hall base sensor Indium arsenide (InAs) typically, $2 \mathrm{mV} / \mathrm{mT}$. The sensitivity of the Hall sensor can be increased by $5 \mathrm{mV} / \mathrm{mT}$ with thin film of Indium Antimonide (InSb) [36]. The frequency of sensitivity for the Hall sensor is ranged from near DC up to $100 \mathrm{kHz}$ [37] with high resolution contribute to higher sensitivity with minimal power consumption.

An early work by Clauzon [38] integrating Hall probe with Eddy current in characterizing a defect under different depth and compared with Finite Element Analysis (FEA). In the observation of the study, they found the correlation between signal characterization and the flaw. Chen [39] investigated a defect classification by integrating Pulse Eddy Current (PEC) which has a pulse excitation providing big frequency information and hall-effect device. The result shows more accurately a 3-dimensional (3D) defect classification compared to the conventional method. A more recent study by Le [40] validated an integration of Dipole Model Method (MDM) using 1024 units of Hall sensor in arrays using alternating current (AC) in estimating the shape and volume of the crack. The time in estimating the crack is proved to be faster and reliable compared to the conventional method by eliminating off-line analysis method.

\subsection{Superconducting Quantum Interference Device (SQUID)}

SQUID sensor known to have an outstanding sensitivity in detecting signal frequency between near DC and low MHz range [41-43]. An experiment done by Faley et al. [44] analysed a spectral density of the SQUID signal which shows a significant decrease of noise value especially in a strong magnetic field as illustrated in Figure 1. The sensor is very useful in detecting a high conductivity material with a deeper defect allocation due to its sensitivity under a low frequency. H. Krause et al. [45] demonstrate a defect scanning using four High Temperature Superconductor (HTS) Direct Current (DC) SQUID that can be operated in a strong magnetic field. The experiment concluded that SQUID has a very excellent performance in detecting a very deep fault. In contrary, in order to achieve a critical temperature, a superconductor in a SQUID has to be cooled off constantly to gain it zero resistance [46].

The main element in the SQUID sensor are the superconducting loop and Radio Frequency (RF) SQUIDs [47] or two DC SQUIDs also known as Josephson junction [48]. The circuit diagram of a dcSQUID sensor comprises of flux transformer and read out electronic [49]. SQUID sensor has a better signal to noise ratio in comparison with a conventional method up to three orders of magnitude for crack exceeding $13 \mathrm{~mm}$ of thickness [50].

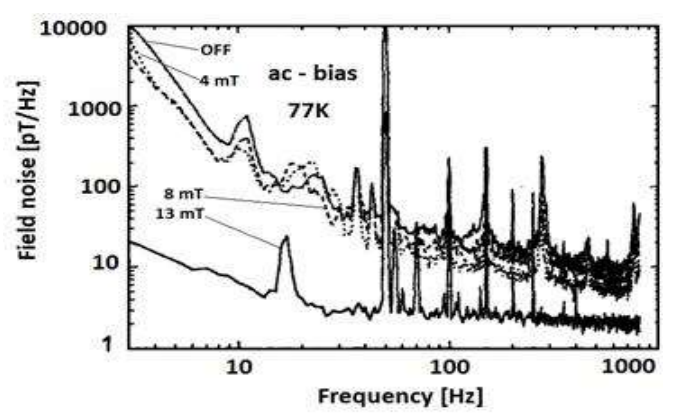

Figure 1. Spectral density of the SQUID sensor using magnetic field up to $13 \mathrm{mT}$ [44]

\subsection{Magnetoresistance (MR) sensor}

The principle magneto resistive sensor is by detecting a change in inductance caused by Eddy current NDE in magnetic field of the specimen [51,52]. The magnetic field will be distributed to the defect properties such as the hole or crack on the subject. The magnetic field is also known for the good capability in testing an ultra-high density magnetic recording in a field of NDE. Tsukada et. al. used MFL technique in detecting a defect in spot welds by MR sensor to investigate the interrelation between the strength and the magnetic measurement of the spot weld [53]. The development of the experiment used two induction coils on both end of the yoke and MR sensor in the middle of the specimen. The output voltage is channel into lock in amplifier before the output data can be obtained.

Int. J. of Adv. in Appl. Sci. Vol. 8, No. 3, September 2019: 208 - 216 
Atzlesberger [54] applied four giant magnetoresistance (GMR) sensors in the experiment to examine a blind hole in ferro magnetic material. However, the sensor likely more sensitive than Anisotropic Magneto Resistor (AMR) due to the design known to have a sensitive axis and nonsensitive axis orthogonally [55]. In the experiment, Helmholtz coil [56] is used by positioning the sensor in the centre of the coil in order to maintain a homogenous magnetic field before the defect properties can be acquired. In order to measure the magnetic flux density on the x-axis between the Helmholtz coil, Biot-Savart's law [57, 58] is used to determine the potential of uncertainty in normal flux distribution. An experiment by Basu et al. [57] by demonstrated an empirical comparison of electromagnetic from the FEA and field computation using the Biot-Savart's law. The field computation is important to ensure the homogeneous condition for the sensor before the measured subject can be acquired.

\section{PRINCIPLE AND INFLUENCE PARAMETER IN MFL DETECTION}

The early work by Sauderson et al. [59] proposed the MFL inspection for above ground storage tanks (ASTs) because of its advantage of being able to cover a large area speedily. At that time, the standard procedure in NDT is by the ultrasonic method which was more tedious and took long time. Qi [60] then explained the advantages of MFL technique for the signal analysis which revealed the correlation between the width of the amplitude of the signal and the defect. It indicates that the MFL signal amplitude varies depend to the width and length of the defect. Furthermore, his finding shows complex relations that interleaves between the variation in of three defect geometries, i.e., width, length and depth of the defects. An analytical function describing the relationship between width, length and signal was carried out by Saha et al. [61] in order to ascertain their depth. The study estimated the depth of the MFL defect in conjunction with the length and width function. Corrosion that was considered as general tended to be oversized in error by less than $5 \%$. However, there are still some ambiguities that can occur. The flux density of a plate in a tank wall can be magnetized using MFL devices [23] The dynamic of an MFL is highly affected by the existence of the defect and detected by hall sensor [62,63]. According to Wang et al. [64], the detection of the signal in MFL does not need a pre-processing. It is because the on-line detection can easily carried out today by implementing high degree of automation. There are several types of defects that can be detected by MFL, for example corrosion pitting, external surface and surface defect [65].

Both circumference and axial direction can be detected by the MFL and it is the most widely used method on ASTs for locating defect on the tank floor, although it is sensitive by other factors [66, 67]. The life of the tank can be increased by repairing the defect includes replacing the entire tank floor [68]. Based on the prevailing damage, individual damaged also can be repaired by welding plates. Product containing impurities in the tank could be the cause of the defect of the tank floor and the reaction between soil and environment is the cause of a defect on the bottom to the tank [69]. Mandache and Clapham, [70] stated that the direct (forward) approach on the main geometry identification which comprises three steps, which are establishing MFL runs, inspection and analysis of the result.

The MFL sensor detects volumetric changes within the leakage field at the corrosion spot [71, 72]. Hence, by measuring the intensity of the flux leakage, the severity of the defect can be determined. The defect of the test piece is detected by measuring the magnetic leakage which making a detour under the defect when magnetic flux passes through the detected region. Figure 2 shows two conditions of MFL sensor in detecting up presence of defect on the test piece. It shows the correlation of the width, amplitude and depth to the defect signal. Both width and amplitude of the signal are proportional to the defect length (axial dimension) but the depth of the defect also affecting the amplitude of the signal [73].

\section{DEFECT GEOMETRIES IN MFL SIGNAL}

Zhang [74] explained the defect geometry parameters such as depth, width and length with their relations between MFL signal features. The vertical component of the defect is unable to be detected by broad and shallow defects and the same result for the defect parallel to the magnetic field. In case of tubes, rods and bars, the longitudinal defect is more easily to be detected with the circular magnetic fields [75]. It is because the magnetic field leaks out from the material if there is any local gradient and geometrical discontinuity in magnetic permeability when the magnetic field applied to a ferromagnetic material.

The three geometric components which is radial, axial and circumferential can be regarded as vectors of the MFL signal [76]. In order to achieve a higher defect sizing capability in pipeline inspection gauges (PIGs), tools for pipeline inspection have also evolved to be able to measure three dimensionsional defects. Typically, only defect geometry that is parallel to the pipeline axis namely axial is being measured [77]. The related work for three dimensionsial defects was done by Xiao-Chun et al. [78] whose optimized the MFL inspection tools for ASTs using finite-element modelling.

Theory and development of magnetic flux leakage sensor for flaws detection: A review (Nor Afandi Sharif) 


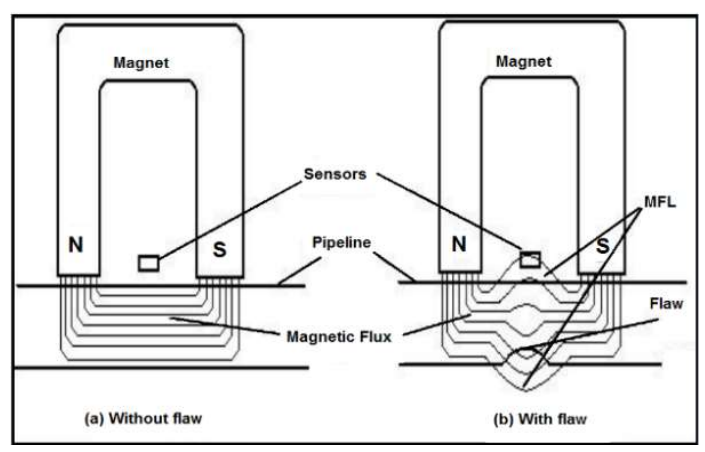

Figure 2. Principle on the MFL signal detection [73]

Feng [79] illustrated three components in the natural cylindrical coordinate named as radial, axial and transverse. All MFL tools are commonly used to measure the axial field components by detecting the disruptions of magnetic field and the volume of the defects. Both traverse and radial field similarly occur when a defect is present and tends to characterize the profile of the feature. Zhang [80] studied the defect allocation on the circumferential component in relative to the sensor spacing, magnetization level and velocity. They study found that crack at the surface of the pipe occurs due to the great difference of pressure, in which the classification wa extremely hard to be seen by the naked eye because the crack is long and very narrow. Garcia [81] further demonstrated the magnitude behaviours between a radial and axial signal. Their research stated that the MFL axial signal's magnitude would be very high compared to the radial signal, and this will happen because the established MFL axial component's direction will parallel the external magneticfield direction.

\section{FEM BACKGROUND IN MFL}

The Finite Element Method (FEM) based simulation is a technique of investigating the behaviours of the affected field in the microscopic level [82]. It began in late 1980's in service inspection of buried pipelines $[72,83]$. In order to achieve an accurate result of field distribution, a full 3-D simulation is then introduced through commercial software. The material property in FEM can be defined as a forward problem and the reconstruction of the crack shape can be defined as the inverse problem in objective to evaluate a parameter in material e.g.; size of the crack, length and depth in the particular field distribution [84, 85].

According to Tupsie et. al. [86], FEM is a general numerical method used for computer simulation. The advantage of FEM compared to others numerical method is the ability to handle circular geometric problem, non-linear and time dependent. It also the most suitable method in solving the issues of magnetic field effect around the transmission line caused by circular cross- section of voltage conductors. There are several software that can characterizes defect by using raw data, including ANSYS, MagNet, JSOL, COMSOL, Multiphysics, OPERA, MAXWELL, FLUX and others [87-89]. These software has their own ability in computing electrostatics and magnetostatics elements. Harmonic and transient problems involving Eddy current can be well for most codes. There are some remarkable tools in implementation of advance function such as a robust solution or hysteresis loop of a moving conductor induction. The functionality of different tools however evolves rapidly and tend to converge [90].

Ji et al. [91] explained the proved of 2-D FEM is an effective method used to study MFL signal under the different material, different defect shape, magnetizing situation and so forth. However, in 2-D FEM defects are furthermore treated as a 2-D profile rather than actual 3-D geometry, and the resulting MFL signal is the single channel whereas the actual signals are multi-channel. The applications of 3D FEM are to analyze and generalize a potential formulation to the magnetic field in MFL and it also accurately modeled and detailed comparison is done for model with defect and without defect [92]. Through FEM, the characteristic of magnetic field intensity and distribution field can be examined. In addition, the FEM can analyse and quantify the distribution of MFL and alteration of the intensity caused by addition of multiple magnetic circuits in order to identify and analyse the generated defect [92]. Similarly to research done by Zakaria [93], Finite Element Method Magnetic (FEMM) is used to model different type of crack. In order to simulate the output in a small section of the pipeline, new properties have been entered into the software in introducing several cracks. A small displacement in the field carries an actual condition of the pipeline as a result from a disturbance of the magnetic field. The simulation presented a SUS 304 steel pipe material and a coil that surround the pipe.

Int. J. of Adv. in Appl. Sci. Vol. 8, No. 3, September 2019: 208 - 216 


\section{APPLICATION ON NEURAL NETWORK ON MFL DATA PROCESSING}

In data mining and clustering, Neural Network (NN) is one of the important tools used as an attempt to build a system that has an ability as a human brain and be capable to learn [94]. Currently, neural network was to use in the field of NDT because its ability to provide a solution in data analysis [95]. Ayad [96] proposed the inversion problem by using the neural Networks for the approximation the mapping from the signal to the defect space. The study points out a crucial problem in signal inversion where the defect profile is recovered from the measured signal by using FEM as an initiator. In tank floor corrosion defect on ASTs, NN also being used to improve accuracy of the defect by establishing Back Propagation Neural Network (BPNN) in order to have a reliable quantitative recognition of width and depth of the defect. Defect in the ASTs can be obtained in three ways, and a lot of MFL testing signal samples are needed in BPNN network [97]. There are three ways of acquiring an MFL testing signal sample of corrosion defect in ASTs in BPNN which need a lot sample:

a. The MFL testing corrosion signal must be extract in the tank floor as a sample testing. Sample taken in the process must be authentic and must maintain close to actual situation of MFL testing corrosion of the tank floor.

b. Artificial neural network is use as precast corrosion defect in the tank floor, a collection of MFL testing signal is recorded in the corrosion database, external factors such as experimental condition and human factor is largely affected.

c. Establishing a finite element as a simulation process to differentiate with the MFL signal collected from the sample of corrosion defect in the tank floor. The interference of numerous detection signals can be avoided and it can solve the problem that the sample number can't meet.

Chen [98] discussed on iterative neural network application on the defect inversion from an MFL signal. There are two kinds of method in solving inverse MFL problems, including model-based iterative method and non-model-based direct method. A non-model based direct method is used to established the relationship between corresponding defect and MFL signal through NN or other tools [99]. Even though the method is advantageous to make a rapid inversion, the prediction of defect profile is not accurate due to lacking of continuous depended of measured MFL signals on defect parameters in non-unique condition. The model-based method on the contrary, use forward model to solve the well-behaved forward problem iteratively in a feedback loop in predicting the MFL signals by begin the algorithm with an initial estimation of the defect and solves the forward problem [100]. The measured and predicted error can be minimised iteratively by updating the defect profile based on gradient-based or optimisation methods [101]

\section{CONCLUSION}

This paper reviews the concept and developments of MFL inspection with regard to principle and influence in detection, defect geometries, FEM and the application of Neural Network in data processing. Several issues need to be improved on such as a noise, defect profiling, development cost, forward model and inverse model. There are a lot of researches has been conducted on MFL signal analysis. However, there are only a few researches emended comprehensive statistical analysis as an inverse model. There is still room for the improvement and optimization of all these issues.

\section{ACKNOWLEDGEMENT}

This work was supported by the The National University of Malaysia (UKM) under research INDUSTRI-2013-010.

\section{REFERENCES}

[1] N. B. Cameron, "Recommended practice for magnetic flux leakage inspection of atmospheric storage tank floors," 2006.

[2] S. Ali and E. Saeedreza, "Intellingent MFL defect detection alghorithm equipped by linear descriminate analysis," IJCNC Int. J. Comput. Networks Commun, vol. 5, pp. 1-21, 2009.

[3] J. Dong, Q. Wu, W. Jiang, and Q. Xu, "The review of new NDT methods of metal material fatigue monitoring," Int. J. Hybrid Inf. Technol., vol. 8, no. 8, pp. 225-232, 2015.

[4] J. Helal, M. Sofi, and P. Mendis, "Non-destructive testing of concrete: A review of methods," Electron. J. Struct. Eng., vol. 14, no. 1, pp. 97-105, 2015.

[5] M. Göktepe, "Investigation of bx and by components of the magnetic flux leakage in ferromagnetic laminated sample," Adv. Mater. Sci. Eng., vol. 2013, 2013.

[6] M. Kollar, V. Setnicka, and R. Zubal, "Scanning and data acquisition tools for MFL testing," 2016.

Theory and development of magnetic flux leakage sensor for flaws detection: A review (Nor Afandi Sharif) 
[7] S. U. N. Liying, et. al., "Comparison of magnetic flux leakage (MFL) and acoustic emission (AE) techniques in corrosion inspection for pressure pipelines," Proc. 31st Chinese Conf., pp. 5375-5378, 2012.

[8] C. F. J. Saavedra and S. R. Prada, "Sensitivity analysis of a magnetic circuit for non- destructive testing by the magnetic flux leakage technique," 2014.

[9] H. M. Kim et. al., "A study on the measurement of axial cracks in the magnetic flux leakage NDT system," IEEE Int. Conf. Autom. Sci. Eng., pp. 624-629, 2012.

[10] J. I. Chang and C. C. Lin, "A study of storage tank accidents," J. Loss Prev. Process Ind., vol. 19, no. 1, pp. 51-59, 2006.

[11] M. R. Kandroodi, B. N. Araabi, and M. N. Ahmadabadi, "Detection of natural gas pipeline defects using magnetic flux leakage measurements," 2013.

[12] M. M. Salama, B. J. Nestleroth, and M. A. Maes, "Characterization of the accuracy of the MFL pipeline inspection tools," Proc. ASME 2012 31st Int. Conf. Ocean. Offshore Arct. Eng., pp. 1-5, 2016.

[13] M. M. Tehranchi, M. Ranjbaran, and H. Eftekhari, "Double core giant magneto-impedance sensors for the inspection of magnetic flux leakage from metal surface cracks," Sensors Actuators, A Phys., vol. 170, no. 1-2, pp. 55-61, 2011.

[14] F. Niese, A. Yashan, and H. Willems, "Wall thickness measurement sensor for pipeline inspection using EMAT technology in combination with pulsed eddy current and MFL," Non-destructive testing, Aust., vol. 3, no. 45, pp. 84-87, 2008.

[15] P. E. Mix, Introduction to nondestructive testing: A training guide, Second Ed. Canada: A John Wiley \& Sons, Inc., Publication, 2005.

[16] X. Wu, J. Xu, and Y. Wang, "Signal processing methods for magnetic flux leakage testing based on biospectrum," Proceeding Eur. NDT Conf., 2010.

[17] H. Krause and M. Kreutzbruck, "Recent developments in SQUID NDE," Phys. C, vol. 368, pp. 70-79, 2002.

[18] A. Sophian, G. Y. Tian, and S. Zairi, "Pulsed magnetic flux leakage techniques for crack detection and characterisation," Sensors and Actuators, vol. 125, pp. 186-191, 2006.

[19] D. Perin et. al., "Mass effect on magnetic flux behavior to cite this article mass effect on magnetic flux behavior," 2017.

[20] Y. Ma, R. He, and J. Chen, "A method for improving SNR of drill pipe leakage flux testing signals by means of magnetic concentrating effect," IEEE Trans. Magn., vol. 51, no. 9, 2015.

[21] M. Yilai, L. Li, J. Kaiwen, and Z. Xulin, "The application of magnetic shielding effect in drill pipe magnetic leakage flux testing," Fifth Conf. Meas. Technol. Mechatronics Autom., no. 1, pp. 1135-1138, 2013.

[22] T. Izgi, M. Goktepe, N. Bayri, V. S. Kolat, and S. Atalay, "Crack detection using fluxgate magnetic field sensor," Acta Phys. Pol. A, vol. 125, no. 2, pp. 211-213, 2014.

[23] H. Can et. al., "Optimizing the sensing performance of a single-rod fluxgate magnetometer using thin magnetic wires," Meas. Sci. Technol., vol. 26, no. 11, 2015.

[24] Z. Zhaoming, S. Taihe, Y. Runqiao, and W. Fu, "A new approach to in-service coiled tubing improved inspection based micro-magnetic technology," Soc. Pet. Eng. - Coiled Tubing Well Interv. Conf. Exhib. 2015, pp. 1-13, 2015.

[25] F. Vacher, F. Alves, and C. Gilles-Pascaud, "Eddy current nondestructive testing with giant magneto-impedance sensor," NDT E Int., vol. 40, no. 6, pp. 439-442, 2007.

[26] W. Dehui, S. Lingxin, W. Xiaohong, and L. Zhitian, "A novel non-destructive testing method by measuring the change rate of magnetic flux leakage," J. Nondestruct. Eval., vol. 36, no. 2, pp. 24, 2017.

[27] K. Mohri, T. Uchiyama, L. P. Shen, C. M. Cai, and L. V. Panina, "Sensitive micro magnetic sensor family utilizing magneto-impedance (MI) and stress-impedance (SI) effects for intelligent measurements and controls," Sensors Actuators A Phys., vol. 91, no. 1-2, pp. 85-90, 2001.

[28] N. Bayri and S. Atalay, "Giant stress-impedance effect in Fe71Cr7Si 9B13 amorphous wires," J. Alloys Compd., vol. 381, no. 1-2, pp. 245-249, 2004.

[29] K. Mohri, T. Uchiyama, L. P. Shen, C. M. Cai, and L. V. Panina, "Amorphous wire and CMOS IC-based sensitive micro-magnetic sensors (MI sensor and SI sensor) for intelligent measurements and controls," J. Magn. Magn. Mater., vol. 249, no. 1-2, pp. 351-356, 2002.

[30] H. M. Kim and G. S. Park, "A study on the estimation of the shapes of axially oriented cracks in CMFL Type NDT system," IEEE Trans. Magn., vol. 50, no. 2, pp. 18-21, 2014.

[31] L. Ben Gur, E. Tirosh, A. Segal, G. Markovich, and A. Gerber, "Extraordinary hall-effect in colloidal magnetic nanoparticle films," J. Magn. Magn. Mater., vol. 426, pp. 178-182, 2017.

[32] R. Clark, "Rail flaw detection: Overview and needs for future developments," NDT E Int., vol. 37, no. 2, pp. 111-118, 2004.

[33] Parker Compumotor Division Hannifin Corporation, "Hall effect sensors," Compumotor - Servo Drive User Guide, pp. 107-118, 2000.

[34] K. Kosmas, C. Sargentis, D. Tsamakis, and E. Hristoforou, "Non-destructive evaluation of magnetic metallic materials using hall sensors," J. Mater. Process. Technol., vol. 161, no. 1-2, pp. 359-362, 2005.

[35] Y. Lijian, L. Gang, G. Songwei, and A. Coil, "Sensor development and application on the oil-gas pipeline magnetic flux leakage detection," Ninth Int. Conf. Electron. Meas. Instruments, pp. 876-878, 2009.

[36] P. Ripka and M. Janosek, "Advances in magnetic field sensors," IEEE Sens. J., vol. 10, no. 6, pp. 1108-1116, 2010.

[37] D. G. Park, et. al., "Evaluation of pulsed eddy current response and detection of the thickness variation in the stainless steel," IEEE Trans. Magn., vol. 45, no. 10, pp. 3893-3896, 2009.

Int. J. of Adv. in Appl. Sci. Vol. 8, No. 3, September 2019: 208 - 216 
[38] T. Clauzon, F. Thollon, and A. Nicolas, "Flaws characterization with pulsed eddy currents N.D.T.," IEEE Trans. Magn., vol. 35, no. 3, pp. 1873-1876, 1999.

[39] T. Chen, G. Y. Tian, A. Sophian, and P. W. Que, "Feature extraction and selection for defect classification of pulsed eddy current NDT," NDT E Int., vol. 41, no. 6, pp. 467-476, 2008.

[40] M. Le, J. Lee, J. Jun, J. Kim, S. Moh, and K. Shin, "Hall sensor array based validation of estimation of crack size in metals using magnetic dipole models," NDT E Int., vol. 53, pp. 18-25, 2013.

[41] A. I. Braginski and H. Krause, "Nondestructive evaluation using high-temperature SQUIDs," Phys. C, pp. 179-183, 2000.

[42] M. v. Kreutzbruck et. al., "Defect detection and classification using a SQUID based multiple frequency," lEEE Trans. Appl. Supercond., vol. 11, no. 1, pp. 1032-1037, 2001.

[43] J. R. Maze et. al., "Nanoscale magnetic sensing with an individual electronic spin in diamond," Nature, vol. 455, no. 7213, pp. 644-647, 2008.

[44] F. M. I., P. U., and U. K., "Operation of HTS dc-SQUID sensors in high magnetic fields," IEEE Trans. Appl. Supercond., vol. 9, no. 2, pp. 3386-3391, 1999.

[45] H. Krause, W. Wolf, W. Glaas, E. Zimmermann, and M. I. Faley, "SQUID array for magnetic inspection of prestressed concrete bridges," Phys. C., vol. 368, pp. 91-95, 2002.

[46] A. Lascialfari, T. Mishonov, A. Rigamonti, P. Tedesco, and A. Varlamov, "Superconducting diamagnetic fluctuations in MgB 2," Phys. Rev. B, vol. 65, no. 0, pp. 4-7, 2002.

[47] Y. Zhang, J. Schubert, N. Wolters, M. Banzet, W. Zander, and H. J. Krause, "Substrate resonator for HTS rf SQUID operation," Phys. C Supercond. its Appl., vol. 372-376, no. 1, pp. 282-286, 2002.

[48] Y. Makhlin, G. Schön, and A. Shnirman, "Quantum-state engineering with josephson-junction devices," Rev. Mod. Phys., vol. 73, p. 357, 2001.

[49] J. Vrba and S. E. Robinson, "SQUID sensor array configurations for magnetoencephalography applications," Supercond. Sci. Technol., vol. 15, no. 9, 2002.

[50] P. Gao, C. Wang, Y. Li, and Z. Cong, "Electromagnetic and eddy current NDT in weld inspection: A review," Insight Non-Destructive Test. Cond. Monit., vol. 57, no. 6, pp. 337-345, 2015.

[51] K. Tsukada, T. Kiwa, T. Kawata, and Y. Ishihara, "Low-frequency eddy current imaging using mr sensor detecting tangential magnetic field components for nondestructive evaluation," IEEE Trans. Magn., vol. 42, no. 10, pp. 3315-3317, 2006.

[52] C. S. Angani, H. G. Ramos, A. L. Ribeiro, and T. J. Rocha, "Evaluation of transient eddy current oscillations response for thickness measurement of stainless-steel plate," Meas. J. Int. Meas. Confed., vol. 90, pp. 59-63, 2016.

[53] K. Tsukada, M. Yoshioka, T. Kiwa, and Y. Hirano, "A magnetic flux leakage method using a magnetoresistive sensor for nondestructive evaluation of spot welds," NDT E Int., vol. 44, no. 1, pp. 101-105, 2011.

[54] J. Atzlesberger and B. Zagar, "Magnetic flux leakage measurement setup for defect detection," Procedia Eng., vol. 5, pp. 1401-1404, 2010.

[55] Y. Kataoka and O. Shinoura, "65.2: Leakage flux testing using a GMR line sensor and a rotating magnetic field," IEEE, pp. 800-803, 2002.

[56] J. Lee, J. Hwang, J. Jun, and S. Choi, "Nondestructive testing and crack evaluation of ferromagnetic material by using the linearly integrated hall sensor array," J. Mech. Sci. Technol., vol. 22, no. 12, pp. 2310-2317, 2008.

[57] S. Basu, S. S. Pany, P. Bannerjee, and S. Mitra, "Pulsed magnetic field measurement outside finite length solenoid: Experimental results \& mathematical verification," J. Electromagn. Anal. Appl., pp. 371-378, 2013.

[58] C. Udri and S. Udri, "Biot-savart-laplace dynamical systems," Balk. J. Geom. Its Appl., vol. 1, no. 2, pp. 125-136, 1996.

[59] D. H. Sauderson, "The MFE tank floor scanner - a case history," IEE Colloq. non-destructive Eval., vol. 1, 1988.

[60] J. Qi, "Experimental study of interference factors and simulation on oil-gas pipeline magnetic flux leakage density signal," Int. Conf. Mechatronics Autom., pp. 3652-3656, 2007.

[61] S. Saha, S. Mukhopadhyay, U. Mahapatra, S. Bhattacharya, and G. P. Srivastava, "Empirical structure for characterizing metal loss defects from radial magnetic flux leakage signal," NDT E Int., vol. 43, no. 6, pp. 507-512, 2010.

[62] R. K. Amineh, et. al., "A space mapping methodology for defect characterization from magnetic flux leakage measurements," IEEE Trans. Magn., vol. 44, no. 8, pp. 2058-2065, 2008.

[63] S. Mukhopadhyay and G. P. Srivastava, "Characterization of metal loss defects from magnetic flux leakage signals with discrete wavelet transform," NDT E Int., vol. 33, no. 1, pp. 57-65, 2000.

[64] Y. Wang, Y. Xu, B. Wang, S. Ding, J. Xu, and M. Zheng, "Research on metal atmospheric storage tank inspection method for standard in China," Proc. ASME 2009 Press. Vessel. Pip. Div. Conf., pp. 1-6, 2017.

[65] N. Pearson, "Discrimination of top and bottom discontinuities with MFL and the surface topology air-gap reluctance system (STARS)," Non-Destructive Testing - Australia, vol. 49(2), pp. 48-51, 2012.

[66] L. Chen, X. Li, X. Li, and Z. Huang, "Signal extraction using ensemble empirical mode decomposition and sparsity in pipeline magnetic flux leakage nondestructive evaluation," Rev. Sci. Instrum., vol. 80, no. 2, p. 25105, 2009.

[67] N. R. Pearson, M. A. Boat, R. H. Priewald, M. J. Pate, and J. S. D. Mason, "A study of MFL signals from a spectrum of defect geometries," 18th World Conf. Nondestruct. Test., pp. 16-20, 2012.

[68] N. R. Pearson, J. S. D. Mason, and R. H. Priewald, "The influence of maintenance on the life cycle of above-ground storage tanks," Insight Non-Destructive Test. Cond. Monit., vol. 54, no. 6, pp. 311-315, 2012.

[69] J. S. D. Mason, N. Pearson, and A. R. Ramırez, "Experimental study to differentiate between top and bottom defects for MFL tank floor inspections," NDT\&E Int., vol. 42, pp. 16-21, 2009.

[70] C. Mandache and L. Clapham, "A model for magnetic flux leakage signal," J. Phys. D. Appl. Phys., vol. $2427,2003$.

Theory and development of magnetic flux leakage sensor for flaws detection: A review (Nor Afandi Sharif) 
[71] W. T. Ming, "Master of science (Physic)," Universiti Teknologi Malaysia, 2004.

[72] K. Mandal and D. L. Atherton, "A study of magnetic flux-leakage signals," J. Phys. D. Appl. Phys., vol. 31, pp. 3211-3217, 1999.

[73] S. Nagu, "Finite element modeling of magnetic flux leakage technique in plates with defect and without defect," Int. J. Mod. Eng. Res., vol. 3, pp. 3452-3455, 2013.

[74] Z. Guoguang, "Defect quantitative recognition technology of circumferential magnetic flux leakage inspection in pipeline," Int. Conf. Comput. Appl. Syst. Model. (ICCASM), pp. 311-314, 2010.

[75] Y. Li, J. Wilson, and G. Y. Tian, "Experiment and simulation study of 3D magnetic field sensing for magnetic flux leakage defect characterisation," NDT E Int., vol. 40, no. 2, pp. 179-184, 2007.

[76] S. M. Dutta, F. H. Ghorbel, and R. K. Stanley, "Dipole modeling of magnetic flux leakage," IEEE Trans. Magn., vol. 45, no. 4, pp. 1959-1965, 2009.

[77] G. Kopp and H. Willems, "Sizing limits of metal loss anomalies using tri-axial MFL measurements: A model study," NDT E Int., vol. 55, pp. 75-81, 2013.

[78] X. Song, S. Huang, and W. Zhao, "Optimization of the magnetic circuit in the MFL inspection system for storagetank floors," Russ. J. Nondestruct. Test., vol. 43, no. 5, pp. 326-331, 2007.

[79] F. Qingshan, J. Sutherland, B. Gu, Y. Wei, and C. Tao, "Evolution of Triax Magnetic Flux Leakage Inspection for Mitigation of Spiral Weld Anomalies," 6th Pipeline Technol. Conf., 2011.

[80] Z. Guoguang and L. Penghui, "Signal processing technology of circumferential magnetic flux leakage inspection in pipeline," Third Int. Conf. Meas. Technol. Mechatronics Autom. Signal, no. 5, pp. 5-8, 2011.

[81] D. A. T. Garcia, "Modelling and interpetation of magnetic flux leakge," RICE University, Houston, Texas, 2012.

[82] Y. Li, G. Y. Tian, and S. Ward, "Numerical simulation on magnetic flux leakage evaluation at high speed," NDT E Int., vol. 39, no. 5, pp. 367-373, 2006.

[83] D. L. Atherton and M. G. Daly, "Finite element calculation of magnetic flux leakage detector signals," NDT Int., vol. 20, no. 4, pp. 235-238, 1987.

[84] M. Li and D. A. Lowther, "The application of topological gradients to defect identification in magnetic flux leakagetype NDT," IEEE Transactions on Magnetics, vol. 46, no. 8, pp. 3221-3224, 2010.

[85] H. Tsuboi, N. Seshima, I. Sebestyen, J. Pavo, S. Gyimothy, and A. Gasparics, "Transient eddy current analysis of pulsed eddy current testing by finite element method," IEEE Trans. Magn., vol. 40, no. 2, pp. 1330-1333, 2004.

[86] S. Tupsie, A. Isaramongkolrak, and P. Pao-la-or, "Analysis of electromagnetic field effects using FEM for transmission lines transposition," Eng. Technol., vol. 3, no. 2, pp. 870-874, 2009.

[87] J. Daniel and R. M. Abudhahir, "Characterization of defects in magnetic flux leakage (MFL) Images using wavelet transform and neural network," 2014 Int. Conf. on Electronics and Communication Systems (ICECS), 2014.

[88] W. Zhang, Y. B. Shi, and Y. J. Li, "Magnetic coil parameters design of oil casing damage detector based on magnetic flux leakage," Proc. 2011 IEEE Int. Conf. Appl. Supercond. Electromagn. Devices, pp. 50-53, 2011.

[89] W. S. Singh, B. P. C. Rao, S. Thirunavukkarasu, and T. Jayakumar, "Flexible GMR sensor array for magnetic flux leakage testing of steel track ropes," J. Sensors, vol. 2012, pp. 1-6, 2012.

[90] M. Augustyniak and Z. Usarek, "Finite element method applied in electromagnetic NDTE - a Review," J. Nondestruct. Eval., pp. 1-16, 2016.

[91] F. Ji, C. Wang, S. Sun, and W. Wang, "Application of 3-D FEM in the simulation analysis for MFL signals," Insight Non-Destructive Test. Cond. Monit., vol. 51, no. 1, pp. 32-35, 2009.

[92] G. Ting-yan, Y. Yong-liang, and H. Tian-yu, "The interaction of multiple magnetic circuits in magnetic flux leakage (MFL) inspection," 2014 IEEE Far East Forum on Nondestructive Evaluation/Testing, 2014.

[93] Z. Zakaria et. al., "Simulation of magnetic flux leakage (MFL) analysis using FEMM software," 2010 IEEE Symp. Ind. Electron. Appl. (ISIEA 2010), pp. 481-486, 2010.

[94] S. W. Lin, K. C. Ying, S. C. Chen, and Z. J. Lee, "Particle swarm optimization for parameter determination and feature selection of support vector machines," Expert Syst. Appl., vol. 35, no. 4, pp. 1817-1824, 2008.

[95] D. Y. Singh and A. S. Chauhan, "Neural networks in data mining," J. Theor. Appl. Inf. Technol., vol. 5, no. 1, pp. 1-154, 2009.

[96] A. Abdelgahani, et. al., "Solution of inverse problems in electromagnetic NDT using neural networks," Przeglad Elektrotechniczny (Electrical Rev.), vol. 87, no. 9, pp. 330-333, 2011.

[97] Z. Yang, G. Dai, W. Li, and Y. Jiang, "Research on magnetic flux leakage signals quantity technology of tank floor corrosion defects based on artificial neural network," Fifth Int. Conf. Nat. Comput. Res., 2009.

[98] J. Chen, S. Huang, and W. Zhao, "Three-dimensional defect inversion from magnetic flux leakage signals using iterative neural network," IET Sci. Meas. Technol., vol. 9, pp. 418-426, 2015.

[99] Y. Zhang, Z. Ye, and C. Wang, "A fast method for rectangular crack sizes reconstruction in magnetic flux leakage testing," NDT E Int., vol. 42, no. 5, pp. 369-375, 2009.

[100] P. Ramuhalli, L. Udpa, and S. S. Udpa, "Neural network-based inversion algorithms in magnetic flux leakage nondestructive evaluation," J. Appl. Phys., vol. 93, no. 10, pp. 8274-8276, 2003.

[101] H. Wenhua and Q. Peiwen, "2-D defect reconstruction from MFL signals based on genetic optimization algorithm," IEEE Int. Conf. Ind. Technol., pp. 508-513, 2005. 